\title{
Efecto de la motivación del entrenador sobre la carga interna y el rendimiento físico de un juego de fútbol reducido
}

\author{
Effect of coach motivation in training load and \\ physical performance of small sided games
}

\section{Efeito motivação do treinador em carga de treinamento e desempenho físico de pequenos jogos lados}

Javier Sánchez-Sánchez , Jesús Ma Luis Pereira, Javier Guillen Rodríguez², David Martín García², Daniel Romo Martín², Alejandro Rodríguez Fernández ${ }^{3}$, José Gerardo Villa Vicente ${ }^{3}$

1 Facultad de Educación. Universidad Pontificia de Salamanca, 2 Club Deportivo Salmantino, 3 Instituto de Biomedicina. Universidad de León.

Resumen: Los juegos reducidos de fútbol son una estrategia de entrenamiento muy empleada en la actualidad. Manipulando sus parámetros configuradores, el entrenador puede conseguir diferentes propósitos. El objetivo del trabajo fue analizar la influencia de la motivación del entrenador sobre la intensidad de un juego reducido (JR) de 3 contra 3 y su efecto sobre el rendimiento físico de jugadores de categoría alevín. Doce futbolistas realizaron el mismo juego reducido con motivación del entrenador (JME) y sin motivación del técnico (JSME). La carga interna de cada tarea fue cuantificada mediante el registro de la frecuencia cardíaca. Antes y después del JR los jugadores realizaron un test de velocidad (sprint de $30-\mathrm{m}$ ), un test de fuerza explosiva de piernas (test triple Hop) y un test de agilidad (test de Illinois). La participación motivante del entrenador provoca una frecuencia cardíaca media, expresada como porcentaje de la frecuencia cardíaca máxima, significativamente mayor que la obtenida sin la motivación del entrenador $(89.12 \pm 4.29 \%$ vs. $82.15 \pm 3.12 \%$ respectivamente). Además, se ha observado una pérdida significativa de rendimiento en el test de velocidad $(5.15 \pm 0.21$ s pre-test y $5.43 \pm 0.27$ s post-test), agilidad $(20.25 \pm 0.86$ s pre-test y $21.01 \pm 0.99$ s post-test $)$ y salto con pierna hábil $(4.88 \pm 0.43 \mathrm{~m}$ pre-test y $4.58 \pm 0.41 \mathrm{~m}$ post-test $)$ tras realizar el JME. El JSME sólo provoca un descenso significativo del rendimiento en el test de velocidad $(5.28 \pm 0.27$ s pre-test y $5.51 \pm 0.22$ s post-test). La motivación del entrenador aumenta la intensidad del JR 3 contra 3.

Palabras clave: Juegos de fútbol reducido; refuerzo positivo; frecuencia cardíaca; velocidad; fuerza explosiva.

Abstract: Small sided games are a training strategy widely used currently. Manipulating their parameters, the coach can achieve different purposes. The aim was to analyze the influence of coach motivation on the intensity of small sided games of 3 vs. 3 and their effect on physical performance in youth male football players (U11). Twelve players made the same game with (JME) and without (JSME) coach motivation. The internal load of each task was quantified by recording heart rate. Before and after small sided games, soccer players perform a speed test (sprint 30-m), explosive strength (triple Hop test) and agility test (Illinois test). Small sided games with coach motivation get a greater mean hear rate, expressed as percentage of $\mathrm{HR}_{\max }(89.12 \pm 4.29 \%$ vs. $82.15 \pm 3.12 \%)$. In addition small sided games with coach motivation significantly decreases performance in sprint test $(5.15 \pm 0.21 \mathrm{~s}$ pre-test and $5.43 \pm 0.27$ s post-test) agility tests $(20.250 .86$ s pre-test and $21.01 \pm 0.99$ s post-test $)$ and jump test $(4.88 \pm 0.43 \mathrm{~m}$ pre-test and $4.58 \pm 0.4 \mathrm{~m}$ post-test). Small sided games without coach motivation only significantly decrease performance in sprint test $(5.28 \pm 0.27 \mathrm{~s}$ pre-test and $5.51 \pm 0.22 \mathrm{~s}$ post-test $)$. Motivation coach increases intensity in small sided games of 3 vs. 3 .

Key words: Small Sided Games; coach encouragement; hear rate; speed; explosive strength.

Resumo: Jogos de futebol reduzida são uma estratégia de treinamento muito usado hoje. Manipulando seus parâmetros configurators, o treinador pode obter efeitos diferentes. O objetivo foi analisar a influência da motivação do treinador da intensidade de um jogo pequeno (JR) $3 \mathrm{em} \mathrm{3}$, e seu efeito sobre o desempenho físico dos jogadores na categoria júnior. Doze jogadores fizeram o mesmo jogo pequeno, treinador motivado (JME) sem a motivação do técnico (JSME). A carga interna de cada tarefa foi quantificada através da gravaçáo de freqüência cardíaca. Antes e depois de jogadores JR realizado um teste de velocidade (sprint de 30-m), um teste de força explosiva perna (test triple Hop) e um teste de agilidade (test de Illinois). A participação treinador motivador causa uma frequência cardíaca média, expresso em percentagem da frequência cardíaca máxima, significativamente mais elevada do que a obtida sem a motivação do treinador $(89.12 \pm 4.29 \%$ vs. $82.15 \pm 3.12 \%$ respectivamente). Além disso, verificou-se uma perda significativa de desempenho no teste de velocidade $(5.15 \pm 0.21 \mathrm{~s}$ pre-test e $5.43 \pm 0.27$ s post-test $)$, agilidade $(20.25 \pm 0.86$ s pre-test e $21.01 \pm 0.99$ s post-test) e saltar com a perna de trabalho $(4.88 \pm 0.43 \mathrm{~m}$ pre-test e $4.58 \pm 0.41$ $\mathrm{m}$ post-test) depois JME. O JSME só provoca uma queda significativa no desempenho em teste de velocidade $(5.28 \pm 0.27 \mathrm{~s}$ pre-test e $5.51 \pm 0.22 \mathrm{~s}$ post-test). A motivação do treinador, aumenta a intensidade do JR de 3 em 3.

\section{Introducción}

Futbolistas de todas las edades entrenan en diferentes escuelas

Dirección para correspondencia [Correspodence address]: Javier Sánchez Sánchez. Facultad de Educación. Universidad Pontificia de Salamanca. C/ Henry Collet 52-70, 37007 Salamanca (España).E-mail: jsanchezsa@upsa.es deportivas con el objetivo de progresar en su deporte (Stroyer, Hansen, y Klausen, 2004). La formación desde los comienzos está articulada en múltiples áreas, puesto que el rendimiento en un deporte como el fútbol depende de factores psicológi- 
cos, sociales, técnicos, tácticos y físicos (Jones y Drust, 2007). Sin perder de vista las demandas del fútbol, los contenidos deberán desarrollarse a través de programas que respeten las características de los deportistas (Capranica, Tessitore, Guidetti, y Figura, 2001). El empleo de juegos reducidos (JRs) permitirá ajustar el entrenamiento al momento evolutivo del niño y a las condiciones del deporte (Rampinini et al., 2007).

Los JRs se han convertido en una forma de trabajo de gran utilidad y atractivo para entrenadores (Radziminski, Rompa, Barnat, Dargiewicz, y Jastrzebski, 2013) y jugadores (Sánchez-Sánchez, Yagüe, y Molinero, 2013). Sobre un clima de alta especificidad (Capranica, et al., 2001; Dellal, Hill-Haas, Lago-Penas, y Chamari, 2011; San Román, Calleja, Castellano y Casamichana, 2010), estas tareas son capaces de mejorar factores básicos del rendimiento deportivo en jugadores de cualquier edad, género y nivel competitivo (Hill-Haas, Dawson, Impellizzeri, y Coutts, 2011; Katis y Kellis, 2009). Además, la posibilidad de entrenar los contenidos técnicos, tácticos y de condición física de forma simultánea (Owen, Wong del, Paul, y Dellal, 2012), permite incrementar el tiempo útil de sesión (Little y Williams, 2005).

En la etapa de iniciación deportiva se debe insistir en el desarrollo de la técnica y la condición física (Katis y Kellis, 2009). Para que a través de los JRs se puedan estimular estos contenidos, el entrenador debe tener la habilidad de seleccionar correctamente algunas variables importantes (Jones y Drust, 2007): la dimensión del terreno de juego y el número de participantes (Clemente, Couceiro, Matins, y Mendes, 2012), la forma de intervenir sobre el balón (Dellal et al., 2011), la direccionalidad del juego (Aguiar, Botelho, Goncalves, y Sampaio, 2013), la presencia de jugadores comodines (Hill-Haas, Coutts, Dawson, y Rowsell, 2010), la duración y organización de los tiempos de trabajo (Casamichana, Castellano y Dellal, 2013) ó la participación del entrenador durante la tarea (Rampinini et al., 2007). La combinación de estos factores modificará la intensidad de los JRs, tal y como se ha comprobado al estudiar algunos indicadores de la carga como la concentración de lactato (Hill-Haas, Coutts, Rowsell, y Dawson, 2009; Little y Williams, 2005; Rampinini et al., 2007), el esfuerzo percibido por el participante (Dellal et al., 2008), la respuesta de la frecuencia cardíaca (FC) (Katis y Kellis, 2009) ó el número y tipo de esfuerzos realizados (Casamichana y Castellano, 2010; Gabbett y Mulvey, 2008; Hill-Haas et al., 2009).

Con respecto a la intervención del entrenador, en el ámbito del entrenamiento deportivo, se ha demostrado que los deportistas bajo supervisión realizan entrenamientos con una carga más elevada (Mazzetti et al., 2000) y muestran una mayor adherencia al entrenamiento (Coutts, Murphy, y Dascombe, 2004). En el fútbol adulto Rampinini et al. (2007) utilizaron JRs de mantenimiento, con formato de 3, 4, 5 y 6 jugadores, realizados en un espacio de juego pequeño, me- diano y grande. Los autores observaron que las variables FC, ácido láctico y percepción subjetiva del esfuerzo, eran mayores en todas las tareas cuando el entrenador participaba dando ánimos a los jugadores. Otro trabajo con una muestra de jugadores jóvenes, estudio el efecto del entrenador en JRs de 2 contra 2 y 3 contra 3, con orientación del juego y miniporterías (Sampaio et al., 2007). En este trabajo los autores señalan la incidencia significativa de la motivación del entrenador sobre la percepción subjetiva del esfuerzo, pero no sobre los valores de FC.

El objetivo del trabajo fue analizar la influencia de la motivación del entrenador sobre la intensidad de un JR de 3 contra 3 y su efecto sobre el rendimiento físico de jugadores de categoría alevín.

\section{Método}

\section{Diseño experimental}

Un grupo de 12 futbolistas alevines participó en 2 sesiones de entrenamiento que incluían un JR de 3 contra 3 (6 series de 4 min y 30 s recuperación). En una sesión el JR se realizó con motivación del entrenador (JME) y otra sin la motivación del entrenador (JSME). Antes de comenzar el estudio se determinó la composición corporal y la frecuencia cardíaca máxima $\left(\mathrm{FC}_{\max }\right)$ de los jugadores. Los participantes fueron distribuidos en grupos de 3, siguiendo criterios técnicos del entrenador para equiparar el nivel de cada equipo. En cada sesión de entrenamiento se mantuvieron los mismos enfrentamientos, cambiando únicamente la intervención del entrenador. Al comenzar (pre-test) y finalizar (post-test) cada JR los futbolistas realizaron 3 test de condición física, manteniendo el orden de las pruebas en pre-test y post-test de ambas sesiones. La FC fue registrada en el JR de las 2 sesiones de entrenamiento.

\section{Participantes}

Doce futbolistas alevines $(11.33 \pm 0.78$ años de edad, $34.88 \pm$ $8.39 \mathrm{Kg}$ de peso, $140.75 \pm 0.09 \mathrm{~cm}$ de altura, $46.48 \pm 2.45 \mathrm{ml} /$ $\mathrm{Kg} / \mathrm{min}$ de $\mathrm{VO}_{2 \max }$ ), con 3 años de experiencia en el entrenamiento del fútbol, que entrenan 90 minutos 2 veces a la semana (martes y jueves) y disputan un partido cada sábado, participan en un diseńo experimental desarrollando un JME y un JSME. El estudio fue admitido por los responsables técnicos del club. El diseño y protocolo del estudio se ajustó a las normas éticas establecidas en la Declaración de Helsinki, y fue aprobado por el Comité de Ética de la Universidad de León.

Todos los futbolistas presentaron el correspondiente consentimiento informado completado por sus padres o tutores legales. Los futbolistas estaban familiarizados con la práctica de JRs, ya que eran empleados habitualmente en las sesiones de entrenamiento del equipo. 


\section{Procedimiento}

En una sesión de entrenamiento anterior a la intervención se registró el peso (Báscula TANITA* BC-418MA "segmental”, precisión $100 \mathrm{gr}$ ), la talla (Tallímetro Holtex ${ }^{\oplus}$ precisión $1 \mathrm{~cm}$ ) y se determinó la $\mathrm{FC}_{\max }$ de cada jugador mediante el test de Course-Navette (Léger y Lambert, 1982), administrado en el propio terreno de juego con pulsómetros Polar Team System 2 (Polar ${ }^{\oplus}$ Electro Oy, Finland).

Los 12 participantes fueron divididos en 4 equipos de 3 futbolistas, según el criterio del técnico, quien organizó equipos homogéneos valorando la demarcación, la competencia técnico-táctica, la aptitud física y la participación en la competición de los jugadores (Casamichana y Castellano, 2009). Para realizar el JR de 3 contra 3 (Owen, Twist, y Fort, 2004) se organizaron 2 espacios de juego de 20 x 15 metros, incluyendo mini-porterías (de 1,5 m de ancho) en los fondos. Para trabajar el principio de la direccionalidad en el juego, cada equipo tenía por objetivo marcar el mayor número de goles en la portería contraria (Sampaio et al., 2007). Los jugadores intervenían sin límite de contactos sobre el balón y podían marcar gol desde cualquier zona del espacio de juego. Para dar continuidad al JR se disponía de balones alrededor del terreno, con el fin de poder reanudar el juego inmediatamente cuando la pelota salía fuera del campo (Köklü, Aşçi, Koçak, Alemdaroğlu, y Dündar, 2011).

En la primera sesión de la semana (martes a las 18:00 horas), los jugadores realizaron el JME. En la segunda sesión del microciclo (jueves a las 18:00 horas), los mismos futbolistas, organizados en los mismos equipos, y participando contra idénticos adversarios, ejecutaron el JSME repitiendo el número y tiempo de series de trabajo. En el JME cada campo estaba dirigido por el entrenador y segundo entrenador del equipo, quienes controlaban la actividad, emitían refuerzos positivos ("sigue intentándolo"; "bien hecho"; "buen esfuerzo", etc.) y corregían las acciones técnicas de los jugadores ("sí colocas bien el pie de apoyo, tu próximo golpeo va a ganar en precisión”, etc.) (Rampinini et al., 2007). En el JSME los 2 entrenadores arbitraban la tarea, sin emitir ninguna opinión ni estímulo sobre la acción de los futbolistas. En ambas sesiones, los jugadores efectuaron 15 minutos de calentamiento $(2 \mathrm{~min}$ de carrera de baja intensidad, 5 min de movilidad articular, 3 min de juego de activación y 5 min de acciones técnicas simples por parejas), e inmediatamente después realizaron los test físicos iniciales. Posteriormente desarrollaron el JR de 3 contra 3, utilizando un método interválico, con 6 series de 4 minutos de trabajo y 90 segundos de recuperación entre cada secuencia. Durante la recuperación los jugadores realizaron estiramientos de baja intensidad y se hidrataron. Tras el JR los jugadores repitieron los test físicos en el mismo orden que durante el pre-test.

Para analizar el efecto de la carga física del JME y del JSME sobre el rendimiento condicional de los jugadores, se administraron 3 test específicos de velocidad, fuerza explosiva y agilidad. El orden de las pruebas en la intervención con el JME y el JSME, se determinó de forma aleatoria para el pre-test (Katis y Kellis, 2009) y se mantuvo en el post-test: Test de sprint 30-m, Test Triple Hop y Test Illinois. Con el objeto de homogeneizar el tiempo que transcurre desde el final del JME y del JSME y el comienzo del post-test, en un campo de juego se comenzó 8 minutos más tarde que en otro. Todos los test se realizaron en el campo de hierba artificial donde semanalmente entrenaban los jugadores. Los futbolistas realizaron las pruebas con el calzado y la indumentaria de entrenamiento habitual. Los test empleados formaban parte del protocolo de evaluación que el club administraba a sus diferentes equipos, por lo que los futbolistas estaban familiarizados con su desarrollo.

\section{Test de condición física}

El Test de 30-m evalúa la velocidad y aceleración del deportista usando fotocélulas (DSD Láser System ${ }^{\oplus}$, con software Sport Test (v3.2.1)) (Katis y Kellis, 2009). Los participantes realizaron 2 sprints de 30 metros, recuperando 3 minutos entre cada prueba. Se registró el mejor tiempo empleado en recorrer la distancia.

El Test Triple Hop evalúa la fuerza explosiva de la musculatura del miembro inferior (Rösch et al., 2000). El jugador se coloca sobre un apoyo detrás de la línea de inicio, y realiza 3 saltos consecutivos con el pie elegido, intentando alcanzar la mayor distancia posible. El ejercicio se repite con una y otra pierna (pierna hábil y no hábil) de manera consecutiva. El futbolista lo realiza 2 veces, con una recuperación de 2 minutos entre cada intento. Para obtener el resultado se emplea una cinta métrica (precisión 1-cm) extendida en el espacio de ejecución del test. Se registró la mayor distancia conseguida con cada pierna.

El Test de Illinois evalúa la agilidad (Vescovi y McGuigan, 2008). Se dibujan 2 líneas en el terreno de juego a una distancia de $10-\mathrm{m}$. La primera línea sirve de marca de salida y punto de llegada, mientras que la otra línea indica la referencia de giro. El jugador se coloca en el suelo decúbito supino, con las manos a la altura de los hombros (punto A), y a la señal se incorpora y va tan rápido como puede a la marca que está frente a él (punto B), para regresar de nuevo a la referencia de la primera línea (punto C) y comenzar una carrera de ida y vuelta en slalom entre los 4 conos que están separados una distancia de 3,3 m. Finalmente el futbolista se dirige hacia la marca de la segunda línea (punto D) y regresa a la meta (punto E). El jugador tiene 2 intentos para realizarlo correctamente, en caso de que en el primero de ellos derribe un cono, no recorra el camino marcado o se caiga. El tiempo fue registrado con fotocélulas (DSD Láser System ${ }^{\circledast}$, con software Sport Test (v3.2.1)). 
Figura 1. Distribución de las balizas en el Test Illinois

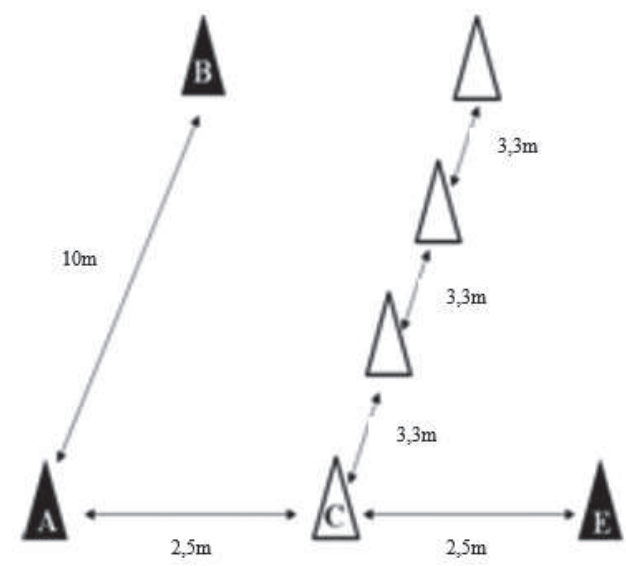

\section{Cuantificación de la carga}

Durante el JME y el JSME se monitorizó la FC de cada jugador, para obtener la carga interna de la tarea. La FC se registró cada 5 segundos usando el equipo Polar Team System 2 (Polar ${ }^{\oplus}$ Electro OY, Finland). Todos los jugadores fueron previamente familiarizados con el uso de este material, y se comprobó que la talla de las bandas se ajustaba a las medidas de los sujetos evaluados. La media de la FC correspondiente a cada serie $\left(\mathrm{FC}_{\text {serie }}\right)$, fue calculada con el software Polar Pro Trainer 5 (Polar ${ }^{\ominus}$ Electro OY). Este valor se expresó en porcentaje $\left(\% \mathrm{FC}_{\operatorname{maxSE}}\right)$ con respecto a la $\mathrm{FC}_{\max }$ de cada jugador obtenida en el Test Course-Navette ( $\mathrm{FC}_{\max }$ Course-Navette):

$\% \mathrm{FC}_{\max \mathrm{SE}}=\left(\mathrm{FC}_{\text {serie }} / \mathrm{FC}_{\max }\right.$ Course-Navette $) \times 100$.

La frecuencia cardiaca media de la tarea, expresada en porcentaje sobre la frecuencia cardíaca máxima individual obtenida en el Test Course-Navette $\left(\% \mathrm{FC}_{\operatorname{maxTOT}}\right)$, se calculó realizando la media de los 6 registros de $\% \mathrm{FC}_{\operatorname{maxSE}}$ :

$$
\% \mathrm{FC}_{\max \mathrm{TOT}}=\% \mathrm{FC}_{\operatorname{maxSE}} / 6 \text {. }
$$

\section{Análisis estadístico}

Se calcularon los estadísticos descriptivos (media \pm desviación estándar) de las variables estudiadas. Se comprobó la normalidad de la muestra a través de la prueba Saphiro-Wilk. A continuación se aplicó la prueba $t$ Student para muestras relacionadas, para comparar la respuesta de la frecuencia cardíaca en cada serie entre el JME y JSME. Para ello se utilizó la media de la FC correspondiente a cada serie de ejercicio, expresada en porcentaje de la frecuencia cardíaca máxima individual $\left(\% \mathrm{FC}_{\max }\right)$. Con el objetivo de constatar el estado inicial de los sujetos en cada día de intervención, esta misma prueba estadística se utilizó para comparar los resultados del pre-test en cada test de condición física. Posteriormente, a fin de evaluar el efecto sobre el rendimiento en test de condición física del JME y el JSME, se compararon lo resultados del pre-test y post-test en cada test de condición física, a través de la prueba $t$ Student para muestras relacionadas. A efectos de interpretación y análisis de los resultados asumimos el 95\% como intervalo de confianza, de manera que se consideró diferencia significativa sí $p \leq .05\left(^{*}\right)$ ó $p \leq .01\left(^{* *}\right)$.

\section{Resultados}

$\mathrm{El} \% \mathrm{FC}_{\operatorname{maxSE}} \mathrm{y} \% \mathrm{FC}_{\max \mathrm{TOT}}$ correspondientes al JME y al JSME, están representados en la Figura 2. El análisis de los resultados indica que en el JME el \% $\mathrm{FC}_{\text {maxTOT }}$ es significativamente mayor $(p \leq .05)$ que en el JSME $(89.12 \pm 4.29 \%$ contra 82.15 $\pm 3.12 \%$, respectivamente). El análisis de la carga interna demuestra un $\% \mathrm{FC}_{\operatorname{maxSE}}$ significativamente mayor en las series 4 y 6 del JME, sin diferencias entre intervenciones en el resto de las series ejecutadas.

Figura 2. Porcentaje de la frecuencia cardíaca máxima en cada serie $\left(\% \mathrm{FC}_{\operatorname{maxSE}}\right)$ y en el juego reducido 3 contra 3 (\% $\left.\mathrm{FC}_{\text {maxTOT }}\right)$, realizado por futbolistas alevines con motivación del entrenador (JME) y sin motivación del entrenador (JSME). Nivel de significación $\left(^{*}\right)(p \leq .05)$.

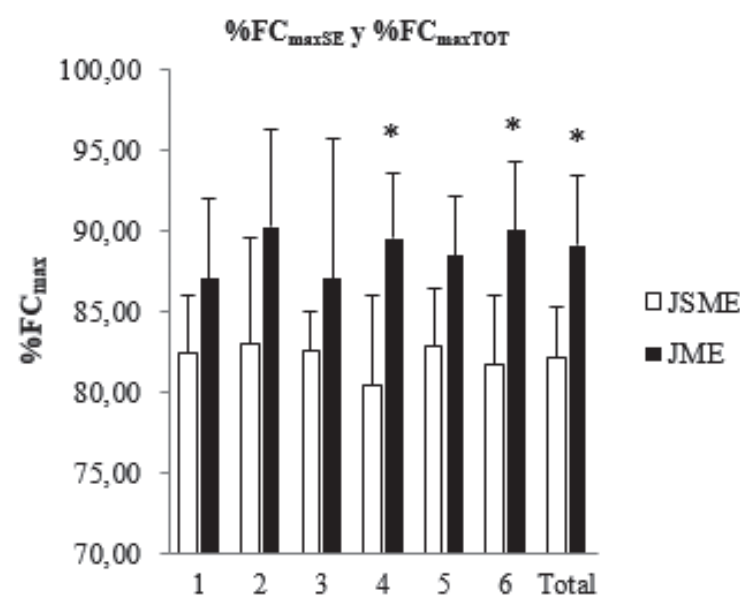

Los resultados obtenidos por ambos grupos en los test de condición física utilizados, muestran que el JME provoca un incremento significativo del tiempo empleado en realizar el test de sprint 30-m y el test de Illinois $(p \leq .01)$. La intervención basada en el JME también empeora de forma significativa los resultados del Test Triple Hop realizado con pierna hábil ( $p$ $\leq .05$ ). La intervención JSME empeora de forma significativa los valores en el test de sprint 30-m ( $p \leq .05)$.

Se obtienen diferencias significativas en el estado inicial de los sujetos en el Test Triple Hop realizado tanto con la pierna hábil como con la no hábil $(p \leq .05)$. Pero no existen diferencias significativas en los resultados del test de sprint 
30-m y del test de Illinois, previo a la realización de las intervenciones. Tampoco se han obtenido diferencias signifi- cativas entre los porcentajes de cambio entre ambos grupos (Tabla 1).

Tabla 1. Test de condición física antes y después de una tarea de juego reducido 3 contra 3 ,

realizada por futbolistas alevines con motivación del entrenador (JME) y sin motivación del entrenador (JSME).

\begin{tabular}{|c|c|c|c|c|c|c|}
\hline & $\mathrm{JME}(\mathrm{n}=12)$ & & & JSME $(n=12)$ & & \\
\hline & Pre-JRs & Post-JRs & $\%$ Per & Pre-JRs & Post-JRs & $\%$ Per \\
\hline Test de Sprint 30-m (s) & $5.15 \pm 0.21$ & $5.43 \pm 0.27^{* *}$ & $-5,32 \pm 3,11$ & $5.28 \pm 0.27$ & $5.51 \pm 0.22 *$ & $-4.62 \pm 6.19$ \\
\hline Test de Illinois (s) & $20.25 \pm 0.86$ & $21.01 \pm 0.99^{* *}$ & $-3,76 \pm 3,32$ & $20.62 \pm 1.15$ & $20.94 \pm 0.95$ & $1.71 \pm 4.53$ \\
\hline Test Triple Hop H (m) & $4.88 \pm 0.43 \dagger$ & $4.58 \pm 0.41^{*} \dagger$ & $-5,82 \pm 7,54$ & $4.20 \pm 0.42$ & $4.13 \pm 0.35$ & $-1.20 \pm 5.09$ \\
\hline Test Triple Hop NH (m) & $4.96 \pm 0.49 \dagger$ & $4.77 \pm 0.57 \dagger$ & $-7,02 \pm 9,33$ & $4.37 \pm 0.41$ & $4.24 \pm 0.43$ & $-4.64 \pm 10.37$ \\
\hline
\end{tabular}

\%Per, Porcentaje de cambio del rendimiento; H, pierna hábil; NH, pierna no hábil.

*Diferencias entre evaluación pre y post JR. $\left({ }^{*}\right)(p \leq .05) ;\left({ }^{* *}\right)(p \leq .01)$

$\dagger$ Diferencias entre grupos $(\dagger)(p \leq .05)$

\section{Discusión}

El objetivo del trabajo fue analizar la influencia de la motivación del entrenador sobre la intensidad de un JR de 3 contra 3 y su efecto sobre el rendimiento físico de jugadores de categoría alevín. En la línea de lo observado en otros trabajos (Rampinini et al., 2007; Sampaio et al., 2007), los resultados muestran que la intervención del entrenador ofreciendo refuerzo positivo y correcciones técnicas a los participantes aumenta la intensidad de la tarea. Los datos de este estudio indican que la respuesta de la FC ha sido un 7.82\% más elevada en el JME que en el JSME. Los valores de 89.12 \pm 4.29 de $\% \mathrm{FC}_{\text {maxTot }}$ observados en el JME son inferiores a los registrados en otro estudio (Köklü et al., 2011) que utiliza un JR de 3 contra 3 incluyendo la intervención del entrenador $\left(92.80 \pm 4.10 \% \mathrm{FC}_{\max }\right)$. El JME utilizado por estos autores, se realiza en un espacio de juego mayor (20x15m vs. 30x18m), lo que nos indica que para valorar la carga interna de una tarea, hay que considerar el conjunto de variables que la configuran (Hill-Haas et al., 2011).

La participación activa del entrenador modifica la carga física de los JRs (Rampinini et al., 2007), ubicándola en umbrales de intensidad similares a los que se registran durante el partido de fútbol (Stolen,Chamari, Castagna, y Wisloff, 2005). Los resultados de la FC en cada una de las series del JME acentúa el valor de la propuesta, ya que no sólo reproduce las demandas técnico-tácticas del juego, sino que además lo consigue con un nivel de exigencia condicional similar a la que reclama la competición (Aguiar et al., 2013). Para conseguir este grado de especificidad los JRs debe ser correctamente diseñado por el entrenador (Hill-Haas et al., 2009). En esta construcción de la tarea se debe tener en cuenta la participación del técnico como emisor de refuerzos positivos hacia los participantes (Rampinini et al., 2007; Sampaio et al., 2007). En otros estudios realizados en contextos deportivos diferen- tes al fútbol, la supervisión del entrenador ha dado grandes resultados respecto al aumento de la carga física de la actividad (Mazzetti et al., 2000), y a una mayor adherencia al entrenamiento por parte de los participantes (Coutts et al., 2004).

Las diferencias de intensidad entre el JME y el JSME pueden ser analizada a través de la FC. Este indicador tradicionalmente se ha empleado en el deporte individual (Achten y Jeukendrup, 2003), pero en la actualidad también se aplica con éxito en disciplinas deportivas para informar de la carga de competición y entrenamiento (Esposito et al., 2004). A pesar de que el registro de la FC es un indicador sencillo, útil y muy empleado (Katis y Kellis, 2009), su sensibilidad frente a elementos como la temperatura, el momento del día y la deshidratación podría restarle precisión en tareas de cuantificación de la carga (Achten y Jeukendrup, 2003). Por este motivo, para categorizar los JRs empleados en el entrenamiento del futbolista, los registros a través de la FC deberían ser completados con otras estrategias como la percepción subjetiva del esfuerzo (Gómez-Díaz, Bradley, Díaz y Pallarés, 2013), las tomas de lactato (Eniseler, 2005) o el análisis de las acciones realizadas durante el juego (Hill-Haas, et al., 2011).

La pérdida de rendimiento en pruebas físicas realizadas antes y después de la participación en JRs, también puede ser empleada para valorar la carga física de la tarea (Katis y Kellis, 2009). En nuestro estudio, la mayor pérdida de rendimiento en los test de condición física, indica una mayor intensidad durante el JME. A pesar de no encontrarse diferencias significativas entre los porcentajes de cambio del rendimiento entre JME y JSME, los resultados obtenidos en los test de sprint, agilidad y salto con la pierna hábil han empeorado significativamente $(-5.32 \pm 3.11 \%,-3.76 \pm 3.32 \%$ y $-5,88 \pm 7.54$ respectivamente) en los jugadores tras la realización del JME. Sin embargo, los test de condición física realizados al finalizar el JSME, demuestran que los futbolistas sólo empeoraron los valores en el test de sprint $(-4.62 \pm 6.9 \%)$. 
En los JRs con bajo número de jugadores se ha descrito la presencia de un gran número de acciones de alta intensidad ejecutadas con balón (Owen et al., 2004). Entre todas estas intervenciones destacan los duelos, que desde el punto de vista técnico-táctico implican la continua realización de regates y entradas (Dellal et al., 2011.). La exigencia que caracteriza a estas acciones, se ve reflejada desde la perspectiva fisiológica en un descenso de las reservas musculares de fosfocreatina (Bangsbo, Iaia, y Krustrup, 2007) y en un aumento de la contribución energética del metabolismo anaeróbico (Dellal et al., 2011). Estos dos factores tienen como consecuencia un aumento en la fatiga del futbolista, que cursa con una reducción del rendimiento en cualidades como la velocidad, la fuerza explosiva y la agilidad (Katis y Kellis, 2009).

A pesar de que nuestro régimen de trabajo es interválico, el tiempo de recuperación junto a un limitado patrón neuromuscular, ha podido limitar el restablecimiento de los sustratos alácticos en los jugadores alevines (Tomlin y Wenger, 2001). Esto puede tener influencia directa sobre la pérdida significativa de rendimiento en el test de velocidad observada tras el JME y el JSME. Los resultados relativos al JME, y en la línea de lo señalado por Katis y Kellis (2009), demuestran que un JR de 3 contra 3, provoca una pérdida de rendimiento en un test de agilidad. La motivación del entrenador durante la tarea, puede incrementar la intervención del jugador, representada por un mayor número de giros, aceleraciones, desaceleraciones y cambios de dirección que pueden fatigar el sistema energético y muscular del que depende la agilidad (Aguiar, M., Botelho, G., Lago, C., Maças, V., y Sampaio, 2012). Por último, aunque se ha observado una reducción de la actividad neuromuscular tras la participación en un partido de fútbol (Mohr, Krustrup, Nybo, Nielsen, y Bangsbo, 2004), al finalizar el JSME, no se observa un peor rendimiento en el test de fuerza explosiva. La intervención del entrenador durante la tarea, tuvo un efecto significativo sobre la pérdida de rendimiento en el Test Triple Hop realizado con la pierna hábil. La mayor intervención sobre el balón que provoca la presencia del técnico y un posible abuso de la pierna hábil por parte de los jugadores, puede ser la causa que explique el comportamiento en este test (Sánchez-Sánchez, Yagüe, Fernández, y Petisco, 2014).
La principal limitación de este estudio fue que se obtuvieron diferencias significativas en el pre-test de la prueba de salto entre los JME y JSME. No ha sido posible controlar la actividad física previa a la realización de las pruebas, realizada por los sujetos que componen la muestra. En el caso de estos futbolistas (11.33 \pm 0.78 años de edad), sus actividades escolares y extraescolares, la heterogeneidad del grupo, elementos técnicos, factores motivacionales y las propias condiciones del test de salto, podrían explicar el diferente rendimiento en cada sesión. Esto no se ha observado en los resultados del test de velocidad y agilidad, donde no se encontraron diferencias significativas.

\section{Conclusión}

La intervención mediante motivación y refuerzos positivos y correcciones por parte del entrenador durante un JR de 3 contra 3 en $20 \times 15$ m, conlleva una mayor intensidad de trabajo en futbolistas alevines. La mayor carga física asociada a las tareas que se realizan acompañadas de la motivación del entrenador, se manifiesta en una pérdida de rendimiento en los test físicos de sprint, agilidad y salto.

\section{Aplicaciones prácticas}

El entrenamiento específico basado en JRs es una estrategia muy usada en la actualidad por los entrenadores de fútbol base. Estos técnicos se preocupan por intervenir sobre variables contextuales de la tarea (espacio, número de jugadores, reglas de intervención, etc), con el propósito de cumplir con los objetivos marcados en la sesión. Entre los aspectos que alteran la respuesta a una tarea, también se debe contemplar la motivación del entrenador, en la medida que su intervención puede condicionar el comportamiento de algunos factores físico-fisiológicos. La participación del entrenador dando refuerzo positivo y controlando la tarea de forma activa, debe ser contemplada en el contexto de formación, como un elemento de gran influencia sobre el joven jugador, que condicionará su respuesta a la actividad de entrenamiento programada.

\section{Referencias}

1. Achten, J., y Jeukendrup, A. E. (2003). Heart rate monitoring: applications and limitations. Sports Medicine, 33(7), 517-538.

2. Aguiar, M. V., Botelho, G. M., Goncalves, B. S., \& Sampaio, J. E. (2013). Physiological responses and activity profiles of football smallsided games. Journal of Strength a Conditioning Research, 27(5), 12871294.

3. Aguiar, M., Botelho, G., Lago, C., Maças, V., y Sampaio, J. (2012). A review on the effects of soccer small-sided games. Journal of Human Kinetics, 33, 103-113.

4. Bangsbo, J., Iaia, F. M., y Krustrup, P. (2007). Metabolic response and fatigue in soccer. International Journal of Sports Physiology and Performance, 2(2), 111-127.

5. Capranica, L., Tessitore, A., Guidetti, L., y Figura, F. (2001). Heart rate and match analysis in pre-pubescent soccer players. Journal of Sports Sciences, 19(6), 379-384.

6. Casamichana, D., y Castellano, J. (2009). Análisis de los diferentes espacios individuales de interacción y los efectos en las conductas motrices de los jugadores: aplicaciones al entrenamiento en futbol. Motricidad. European Journal of Human Movement, 23, 143-167.

7. Casamichana, D., y Castellano, J. (2010). Time-motion, heart rate, 
perceptual and motor behaviour demands in small-sides soccer games: effects of pitch size. Journal of Sports Sciences, 28(14), 1615-1623.

8. Casamichana, D., Castellano, J., y Dellal, A. (2013). Influence of different training regimes on physical and physiological demands during small-sided soccer games: continuous vs. intermittent format. Journal of Strength \& Conditioning Research, 27(3), 690-697.

9. Clemente, F., Couceiro, M. S., Martins, F. M. L., y Mendes, R. U. I. (2012). The usefulness of small-sided games on soccer training. Journal of Physical Education and Sport, 12(1), 93-102.

10. Coutts, A. J., Murphy, A. J., y Dascombe, B. J. (2004). Effect of direct supervision of a strength coach on measures of muscular strength and power in young rugby league players. Journal of Strength \& Conditioning Research, 18(2), 316-323.

11. Dellal, A., Chamari, K., Pintus, A., Girard, O., Cotte, T., y Keller, D. (2008). Heart rate responses during small-sided games and short intermittent running training in elite soccer players: a comparative study. Journal of Strength \& Conditioning Research, 22(5), 1449-1457.

12. Dellal, A., Hill-Haas, S., Lago-Penas, C., y Chamari, K. (2011). Smallsided games in soccer: amateur vs. professional players' physiological responses, physical, and technical activities. Journal of Strength \& Conditioning Research, 25(9), 2371-2381.

13. Eniseler, N. (2005). Heart rate and blood lactate concentrations as predictors of physiological load on elite soccer players during various soccer training activities. Journal of Strength \& Conditioning Research, 19(4), 799-804.

14. Esposito, F., Impellizzeri, F. M., Margonato, V., Vanni, R., Pizzini, G., y Veicsteinas, A. (2004). Validity of heart rate as an indicator of aerobic demand during soccer activities in amateur soccer players. European Journal of Applied Physiology, 93(1-2), 167-172.

15. Gabbett, T. J., y Mulvey, M. J. (2008). Time-motion analysis of smallsided training games and competition in elite women soccer players. Journal of Strength \& Conditioning Research, 22(2), 543-552.

16. Goméz-Díaz A. J., Bradley, P. S., Díaz, A., Pallarés, J. G.(2013). Percepción subjetiva del esfuerzo en fútbol profesional: relevancia de los indicadores físicos y psicológicos en el entrenamiento y la competición. Anales de Psicología, 29(3), 656-661.

17. Hill-Haas, S., Dawson, B., Impellizzeri, F., y Coutts, A. (2011). Physiology of small-sided games training in football: a systematic review. Sports Medicine, 41(3), 199-220.

18. Hill-Haas, S. V., Coutts, A. J., Dawson, B. T., y Rowsell, G. J. (2010). Time-motion characteristics and physiological responses of small-sided games in elite youth players: the influence of player number and rule changes. Journal of Strength \& Conditioning Research, 24(8), 2149-2156.

19. Hill-Haas, S. V., Coutts, A. J., Rowsell, G. J., y Dawson, B. T. (2009). Generic versus small-sided game training in soccer. International Journal of Sports Medicine, 30(9), 636-642.

20. Jones, S., y Drust, B. (2007). Physiological and technical demands of $4 \mathrm{v} 4$ and 8 v 8 games in elite youth soccer players. Kinesiology, 39(2), $150-156$.

21. Katis, A., y Kellis, E. (2009). Effects of small-sided games on physical conditioning and performance in young soccer players. Journal of Sports Science and Medicine, 8(3), 374-380.

22. Köklü, Y., Aşçi, A., Koçak, F. U., Alemdaroğlu, U., y Dündar, U. (2011). Comparison of the physiological responses to different small-sided games in elite young soccer players. Journal of Strength \&Conditioning Research, 25(6), 1522-1528.

23. Léger, L.A., y Lambert, J. (1982). A maximal multistage 20-m shuttle run test to predict VO2max. European Journal of Applied Physiology and
Occupational Physiology, 49(1), 1-12.

24. Little, T., y Williams, A. G. (2005). Specificity of acceleration, maximum speed, and agility in professional soccer players. Journal of Strength $\&$ Conditioning Research, 19(1), 76-78.

25. Mazzetti, S. A., Kraemer, W. J., Volek, J. S., Duncan, N. D., Ratamess, N. A., Gomez, A. L., Fleck, S. J. (2000). The influence of direct supervision of resistance training on strength performance. Medicine \& Science in Sports \& Exercise, 32(6), 1175-1184.

26. Mohr, M., Krustrup, P., Nybo, L., Nielsen, J. J., y Bangsbo, J. (2004). Muscle temperature and sprint performance during soccer matches-beneficial effect of re-warm-up at half-time. Scandinavian Journal of Medicine \& Science in Sports, 14(3), 156-162.

27. Owen, A., Twist, C., y Ford, P. (2004). Small-sided games: the physiological and technical effects of altering pitch size and player numbers. Insight FACA J, 7(2), 50-53.

28. Owen, A. L., Wong del, P., Paul, D., y Dellal, A. (2012). Effects of a periodized small-sided game training intervention on physical performance in elite professional soccer. Journal of Strength \& Conditioning Research, 26(10), 2748-2754.

29. Radziminski, L., Rompa, P., Barnat, W., Dargiewicz, R., y Jastrzebski, Z. (2013). A Comparison of the Physiological and Technical Effects of High-Intensity Running and Small-Sided Games in Young Soccer Players. International Journal of Sports Science and Coaching, 8, 455-466.

30. Rampinini, E., Impellizzeri, F. M., Castagna, C., Abt, G., Chamari, K., Sassi, A., y Marcora, S. M. (2007). Factors influencing physiological responses to small-sided soccer games. Journal of Sports Sciences, 25(6), 659-666.

31. Rösch, D., Hodgson, R., Peterson, L., Graf-BAumann, T., Junge, A., Chomiak, J., y Dvorak, J. (2000). Assessment and evaluation of football performance. American Journal of Sports Medicine, 28(5), 29-39.

32. Sampaio, J., García, G., Maçãs, V., Ibáńez, S., Abrantes, C. y Caixinha, P. (2007). Heart rate and perceptual responses to $2 \times 2$ and $3 \times 3$ small- sided youth soccer games. Journal of Sports Science and Medicine, 6(Supl.10), 121-122

33. San Román, J., Calleja, J., Castellano, J., y Casamichana, D. (2010). Análisis de la capacidad de salto antes, durante y después de la competición en jugadores internacionales junior de baloncesto. RYCIDE. Revista internacional de ciencias del deporte, 6(21), 311-321.

34. Sánchez-Sánchez, J., Yagüe, J. M., Fernández, R. C., y Petisco, C. (2014). Efectos de un entrenamiento con juegos reducidos sobre la técnica y la condición física de jóvenes futbolistas. RICYDE Revista Internacional de Ciencias Del Deporte, 37, 221-234.

35. Sánchez-Sánchez, J., Yagüe, J., y Molinero, O. (2013). Estudio del nivel de diversión generado por la aplicación de un programa de entrenamiento técnico y otro táctico en futbolistas jóvenes. Cuadernos de Psicología Del Deporte, 13(1), 95-102.

36. Stolen, T., Chamari, K., Castagna, C., y Wisloff, U. (2005). Physiology of soccer: an update. Sports Medicine, 35(6), 501-536.

37. Stroyer, J., Hansen, L., y Klausen, K. (2004). Physiological profile and activity pattern of young soccer players during match play. Medicine \& Science in Sports y Exercise, 36(1), 168-174.

38. Tomlin, D. L., y Wenger, H. A. (2001). The relationship between aerobic fitness and recovery from high intensity intermittent exercise. Sports Medicine, 31(1), 1-11.

39. Vescovi, J. D., y McGuigan, M. R. (2008). Relationships between sprinting, agility, and jump ability in female athletes. Journal of Sports Sciences, 26(1), 97-107. 